Elsevier Sequoia S.A., Lausanne - Printed in The Netherlands

\title{
A.C. POLAROGRAPHY WITH SUPERIMPOSED TRIANGULAR VOLTAGE; AN ALTERNATIVE WAY FOR ELIMINATING THE CAPACITY CURRENT
}

\author{
J. H. SLUYTERS, J. S. M. C. BREUKEL AND M. SLUYTERS-REHBACH \\ Laboratory of Analytical Chemistry, State University, Utrecht (The Netherlands) \\ (Received 3rd November 1970)
}

INTRODUCTION

A.c. polarography is a widely used technique, in principle useful for the qualitative and quantitative analysis of substances which are able to undergo an electrode reaction.

In normal sinusoidal a.c. polarography ${ }^{1}$ the sensitivity is in the first place determined by the magnitude of the base line current or capacity current, caused by the existence of the electrical double layer at the electrode-solution interface. In order to suppress the capacity current $i_{\mathrm{C}}$, which is proportional to the frequency, low frequencies are employed. This has, however, the disadvantage that also the faradaic current $i_{\mathrm{F}}$ is lowered, since $i_{\mathrm{F}}$ is proportional to the square root of frequency. A better way of eliminating the capacity current is effected by an a.c. polarograph patented by Jessop $^{2}$, which measures only the in-phase component of the sine wave current. This signal contains theoretically no contribution of the capacity current if the a.c. voltage loss over the ohmic resistance $R_{\Omega}$ is properly compensated ${ }^{3}$. Another, probably more expensive, technique is square-wave a.c. polarography ${ }^{4}$, in which the capacity current is diminished to a large extent by measuring the current at the end of each half period of the applied small amplitude square-wave voltage.

In this paper a new a.c. polarographic technique is proposed, with which theoretically the capacity current is eliminated, so that the base line in the a.c. polarogram is reduced to zero. The principle is the application of a triangular a.c. voltage across the electrode interface, followed by full-wave rectification of the a.c. current. The effect of this on the a.c. polarogram to be obtained will be described in the next section.

THEORY

The polarograph is in principle built according to the block scheme given in Fig. 1. A triangular voltage with frequency $v$ is superimposed on the scanned d.c. voltage and both are applied to a three-electrode cell via a potentiostat. The resulting current is passed through a full-wave rectifier and, after filtering out the d.c. component with a capacitor, fed to an amplifier tuned at the frequency $2 v$. The output of this amplifier is rectified and finally connected to a d.c. recorder. The final result can be understood on the basis of the following considerations. 


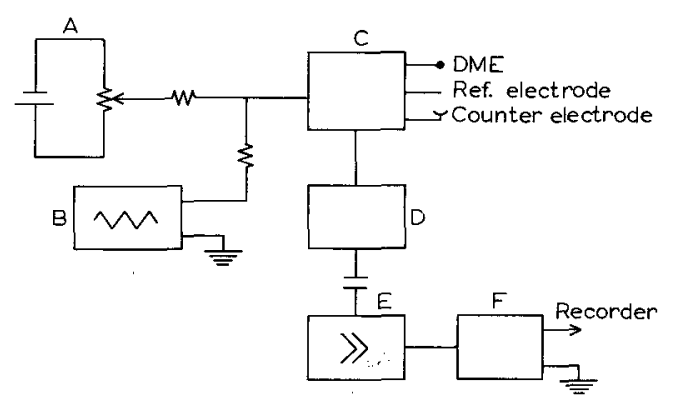

Fig. 1. Block scheme of the apparatus. (A) Ramp generator, (B) triangle generator, (C) potentiostat with current follower amplifier, (D) full wave rectifier, (E) Hewlett-Packard $302 \mathrm{~A}$ as tuned amplifier, (F) integrator.

(a) The potentiostat serves to compensate the $i R$ drop over the ohmic resistance of the cell, consisting of the solution resistance, the resistance of the indicator electrode (usually a dropping mercury electrode) and the wires to the indicator and the counter electrode. If the voltage drop over the total ohmic resistance $R_{\Omega}$ is properly compensated, there results a triangular a.c. voltage $\Delta E$ superimposed on the d.c. voltage $\bar{E}$ across the electrode-solution interface; in other words across the electrical double layer.

(b) The double layer capacity is defined by

$$
C_{\mathrm{d}}=\mathrm{d} q / \mathrm{d} E
$$

where $q$ is the charge density on the electrode surface and $E$ the electrode potential. Consequently the current which charges or discharges the double layer is given by

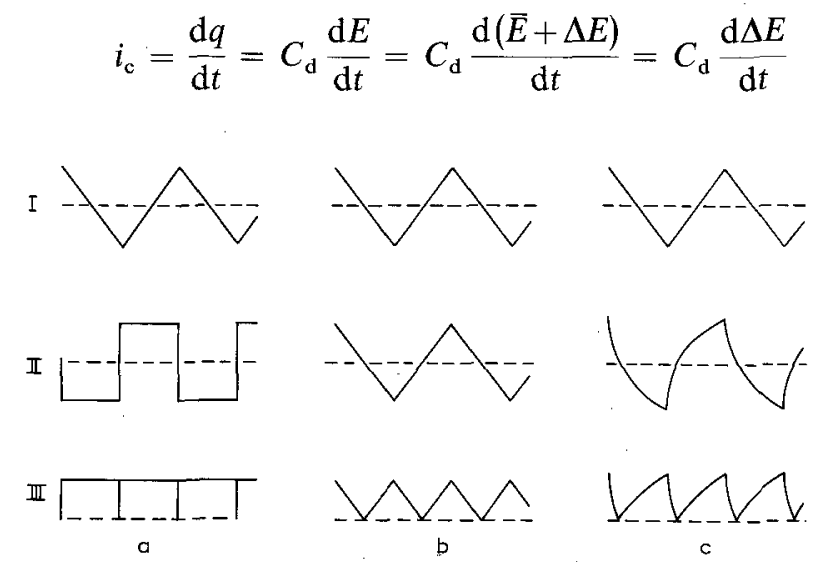

Fig. 2. The various wave forms. (I) Triangular voltage across the interface, (II) resulting current, (III) resultcurrent after rectification. (a) Capacity current, (b) faradaic current for an irreversible system, (c) faradaic current for a reversible system.

The derivative of a triangular wave form is a square wave, so the capacity current will be a square-wave current (Fig. 2a, II). Full-wave rectification of it yields theoretically a d.c. current. In practice spikes will appear at each half period because of unavoidable non-ideality of the triangular and the square-wave form (Fig. 2a, III).

(c) The faradaic current is determined by the current-voltage characteristic 
("absolute rate equation") $)^{5}$

$$
i_{\mathrm{f}}=n F k_{\mathrm{sh}}\left\{c_{\mathrm{R}} \exp \left[\alpha(n F / R T)\left(E-E_{0}\right)\right]-c_{\mathrm{O}} \exp \left[-\beta(n F / R T)\left(E-E_{0}\right)\right]\right\}
$$

in which $c_{\mathrm{O}}$ and $c_{\mathrm{R}}$ are the concentrations of the electroactive species at the electrode surface. If $E$ is the sum of a d.c. polarizing potential $\bar{E}$ and an a.c. voltage $\Delta E$, the current and the concentrations may be written also as a sum of a d.c. component, $\bar{l}, \bar{c}_{\mathrm{R}}$ and $\bar{c}_{\mathrm{O}}$ and an a.c. component $\Delta i, \Delta c_{\mathrm{R}}$ and $\Delta c_{\mathrm{O}}$. One has

$$
\begin{aligned}
\bar{l}+\Delta i=n F k_{\mathrm{sh}}\left\{\left(\bar{c}_{\mathrm{R}}+\Delta c_{\mathrm{R}}\right) \exp (\alpha \varphi) \cdot \exp [\alpha(n F / R T) \Delta E]-\right. \\
\left.-\left(\bar{c}_{\mathrm{O}}+\Delta c_{\mathrm{o}}\right) \exp (-\beta \varphi) \cdot \exp [-\beta(n F / R T) \Delta E]\right\}
\end{aligned}
$$

with

$$
\varphi=(n F / R T)\left(\bar{E}-E_{0}\right)
$$

The exponents of $\Delta E$ can be developed and for small $\Delta E$ and consequently small $\Delta c$ the higher order terms (in $\Delta E^{2}$ and $\Delta E \cdot \Delta c$ ) can be omitted:

$$
\begin{aligned}
\bar{\imath}+\Delta i= & n F k_{\mathrm{sh}}\left\{\bar{c}_{\mathrm{R}} \exp (\alpha \varphi)+(\alpha n F / R T) \bar{c}_{\mathrm{R}} \exp (\alpha \varphi) \Delta E+\Delta c_{\mathrm{R}} \exp (\alpha \varphi)+\right. \\
& \left.-\bar{c}_{\mathrm{O}} \exp (-\beta \varphi)+(\beta n F / R T) \bar{c}_{\mathrm{O}} \exp (-\beta \varphi) \Delta E-\Delta c_{\mathrm{O}} \exp (-\beta \varphi)\right\}
\end{aligned}
$$

In the right hand side of this equation the d.c. component is easily recognized considering eqn. (3). After subtraction of it the a.c. component remains:

$$
\begin{aligned}
\Delta i= & \left(n^{2} F^{2} k_{\mathrm{sh}} / R T\right)\left[\alpha \bar{c}_{\mathrm{R}} \exp (\alpha \varphi)+\beta \bar{c}_{\mathrm{o}} \exp (-\beta \varphi] \Delta E+\right. \\
& n F k_{\mathrm{sh}}\left[\Delta c_{\mathrm{R}} \exp (\alpha \varphi)-\Delta c_{\mathrm{O}} \exp (-\beta \varphi]\right.
\end{aligned}
$$

Equation (7) does not give $\Delta i$ directly as a function of $\Delta E$, because $\Delta c_{\mathrm{R}}$ and $\Delta c_{\mathrm{O}}$ are implicit functions of $\Delta i$, to be found by solving Fick's second law

$$
\frac{\partial \Delta c_{\mathrm{i}}}{\partial t}=D_{\mathrm{i}} \frac{\partial^{2} \Delta c_{\mathrm{i}}}{\partial x^{2}}
$$

with the boundary conditions

$$
\Delta i=-n F D_{\mathrm{O}}\left(\frac{\partial \Delta c_{\mathrm{O}}}{\partial x}\right)_{x=0}=n F D_{\mathrm{R}}\left(\frac{\partial \Delta c_{\mathrm{R}}}{\partial x}\right)_{x=0}
$$

where $D_{\mathrm{O}}$ and $D_{\mathrm{R}}$ are the diffusion coefficients and $x$ is the distance from the electrode surface.

The solution of the problem for a sine wave perturbation is well-known. For an a.c. voltage

$$
\Delta E_{\sim}=\Delta E_{\sim}^{\mathrm{m}} \cos \omega t
$$

one obtains for the current ${ }^{6}$

$$
\Delta i \sim=\frac{\Delta E_{\sim}^{\mathbf{m}}}{\sigma \omega^{-\frac{1}{2}}\left(p^{2}+2 p+2\right)^{\frac{1}{2}}} \cos (\omega t+\phi)
$$




$$
\cot \phi=p+1
$$

in which

$$
\begin{aligned}
& \sigma=\frac{R T}{n^{2} F^{2} \sqrt{2}} \times \frac{D_{\mathrm{R}}^{-\frac{1}{2}} \exp (\alpha \varphi)+D_{\mathrm{O}}^{-\frac{1}{2}} \exp (-\beta \varphi)}{\alpha \bar{c}_{\mathrm{R}} \exp (\alpha \varphi)+\beta \bar{c}_{\mathrm{O}} \exp (-\beta \varphi)} \\
& p=\frac{(2 \omega)^{\frac{1}{2}}}{k_{\mathrm{sh}}} \times \frac{1}{D_{\mathrm{R}}^{-\frac{1}{2}} \exp (\alpha \varphi)+D_{\mathrm{O}}^{-\frac{1}{2}} \exp (-\beta \varphi)}=p^{\prime} \omega^{\frac{1}{2}}
\end{aligned}
$$

Any periodic wave can be written as a Fourier series of sine or cosine terms. Thus, a triangular wave can be written as

$$
\Delta E=E_{\mathrm{m}} \frac{8}{\pi^{2}} \sum_{q=0}^{\infty} \frac{\cos [(2 q+1) 2 \pi v t]}{(2 q+1)^{2}}
$$

in which $E_{\mathrm{m}}$ is the amplitude of the triangular wave and $v$ its frequency.

Relations (10)-(14) can be applied on each term in eqn. (15) considering that

$$
\Delta E_{\sim, q}^{\mathrm{m}}=\frac{8 E_{\mathrm{m}}}{\pi^{2}(2 q+1)^{2}}
$$

and

$$
\begin{aligned}
& \omega_{q}=(2 q+1) 2 \pi v \\
& p_{q}=p^{\prime}(2 q+1)^{\frac{1}{2}}(2 \pi v)^{\frac{1}{2}}
\end{aligned}
$$

Consequently

$$
\Delta i=\frac{8 E_{\mathrm{m}}}{\pi^{2} \sigma(2 \pi v)^{-\frac{1}{2}}} \sum_{q=0}^{\infty} \frac{\cos \left[(2 q+1) 2 \pi v t+\phi_{q}\right]}{(2 q+1)^{\frac{3}{2}}\left(p_{q}^{2}+2 p_{q}+2\right)^{\frac{1}{2}}}
$$

It is convenient to consider the following extreme cases.

(1) For an irreversible electrode process (small $k_{\mathrm{sh}}$ ) $p$ is large. This means that $\phi_{q}$ approaches zero and the term $\left(p_{q}^{2}+2 p_{q}+2\right)^{\frac{1}{2}}$ reduces to $p_{q}$. Equation (17) becomes

$$
\Delta i=\frac{8 E_{\mathrm{m}}}{\pi^{2} \sigma p^{\prime}} \sum_{q=0}^{\infty} \frac{\cos [(2 q+1) 2 \pi v t]}{(2 q+1)^{2}}
$$

thus, considering eqn. (15)

$$
\Delta i=\Delta E / \sigma p^{\prime}
$$

In this case the faradaic current has also the triangular wave form with frequency $v$ and an amplitude $E_{\mathrm{m}} / \sigma p^{\prime}$ (Fig. 2b, II). Full-wave rectification yields a triangular wave with the doubled frequency $2 v$ (Fig. 2b, III).

(2) For a reversible electrode process $k_{\mathrm{sh}}$ is large and $p$ is small. If $p \rightarrow 0, \phi_{q}$ approaches $\pi / 4$ and the term $\left(p_{q}^{2}+2 p_{q}+2\right)^{\frac{1}{2}}$ reduces to $2^{\frac{1}{2}}$. Now eqn. (17) is simplified to

$$
\Delta i=\frac{8 E_{\mathrm{m}}}{\pi^{\frac{3}{2}} \sigma \nu^{-\frac{1}{2}}} \sum_{q=0}^{\infty} \frac{\cos [(2 q+1) 2 \pi v t+\pi / 4]}{(2 q+1)^{\frac{3}{2}}}
$$

The summation term is easily calculated with the aid of a computer. After 300 terms the convergence proved to be sufficient. The values for different values of $v t$ are given in Table 1. 
TABLE 1

THE SUMMATION FACTOR IN EQN. (20) DURING ONE PERIOD TAKING $q=0-300$

\begin{tabular}{llll}
\hline$v t$ & $\Delta i$ & $v t$ & $\Delta i$ \\
\hline 0 & +1.166 & 0.5 & -1.166 \\
0.02 & +0.604 & 0.52 & -0.604 \\
0.04 & +0.380 & 0.54 & -0.380 \\
0.06 & +0.216 & 0.56 & -0.216 \\
0.08 & +0.083 & 0.58 & -0.083 \\
0.1 & -0.031 & 0.6 & +0.031 \\
0.2 & -0.453 & 0.7 & +0.453 \\
0.3 & -0.752 & 0.8 & +0.752 \\
0.4 & -0.992 & 0.9 & +0.992 \\
0.5 & -1.166 & 1.0 & +1.166 \\
\hline
\end{tabular}

The resulting $\Delta i$ has a wave form as depicted in Fig. 2c, II. Evidently full-wave rectification yields a substantial a.c. current with a curved saw-tooth form and also the double frequency $2 v$ (Fig. 2c, III). In this case it is easily possible to indicate the potential dependence of the amplitude since for a reversible system it is known that ${ }^{6}$

$$
\begin{aligned}
\sigma & =\sigma_{\mathrm{m}} \cosh ^{2}\left[(n F / 2 R T)\left(E-E_{\frac{\mathrm{r}}{2}}^{\mathrm{r}}\right)\right] \\
\sigma_{\mathrm{m}} & =\frac{4 R T}{2^{\frac{1}{2}} n^{2} F^{2}\left(c_{\mathrm{O}}^{*} D_{\mathrm{O}}^{\frac{1}{2}}+c_{\mathrm{R}}^{*} D_{\mathrm{R}}^{\frac{1}{2}}\right)}
\end{aligned}
$$

in which $E_{\frac{1}{2}}^{\mathrm{r}}$ is the reversible half-wave potential and $c_{\mathrm{O}}^{*}$ and $c_{\mathrm{R}}^{*}$ are the bulk concentrations.

Of course in the intermediate case between reversible and irreversible, $\Delta i$ can be calculated with the general eqn. (17), in which $p^{\prime}$ should be chosen as a fixed parameter. For our purpose this is not necessary, since it will be clear that the result produces an appreciable ripple with doubled frequency superimposed on the d.c. level caused by the rectified capacity current. In other words, it is theoretically possible, after filtering out the d.c. level, to obtain a faradaic peak in the a.c. polarogram with a zero base line current.

\section{EXPERIMENTAL}

The separate parts of the block circuit in Fig. 1 can be realized in several ways. In the following we will give a more detailed description of our own set-up.

The cell was a usual three-electrode cell with a dropping mercury electrode (DME), a mercury pool counter electrode and a saturated calomel reference electrode. The test solution contained $1 \mathrm{M} \mathrm{KCl}$ as supporting electrolyte and $\mathrm{Cd}^{2+}$ in various concentrations as a reversibly reduced species or $\mathrm{Zn}^{2+}$ as an irreversibly reduced species. The drop-time of the DME was regulated mechanically.

The ramp generator $\mathrm{A}$ was a potentiometer, which was scanned step-wise, triggered by the fall of a mercury drop so that with each drop the d.c. potential shifted by $c a .2 \mathrm{mV}$. For the triangle generator B we used a Philips function generator (PM 5168). However, a triangle generator can easily be built, e.g. in the form of a 
multivibrator with integrator. Its output was attenuated to $10 \mathrm{mV}$ peak to peak.

It is essential that the potentiostat $C$ compensates the total $i R_{\Omega}$ drop also over the resistance in the capillary of the DME and in the vicinity of the mercury drop. We used a potentiostat with positive feedback as designed by Brown et al. ${ }^{7}$ Its circuit contains simultaneously a current follower amplifier, which provides the current passing the electrode-solution interface we are interested in. For the amplifiers we used analog devices $118 \mathrm{~A}$. These are chopper-stabilized amplifiers, which have the disadvantage of a high noise level. Use of low drift chopperless amplifiers, e.g. analog model 184, would be better.

A simple way of full-wave rectification would be to apply a Graetz circuit. This has, however, the disadvantage of earthing problems. Therefore we preferred to use a full-wave rectifier made of operational amplifiers and resistances, described in the Handbook of Burr-Brown ${ }^{8}$. The circuit is drawn in Fig. 3.
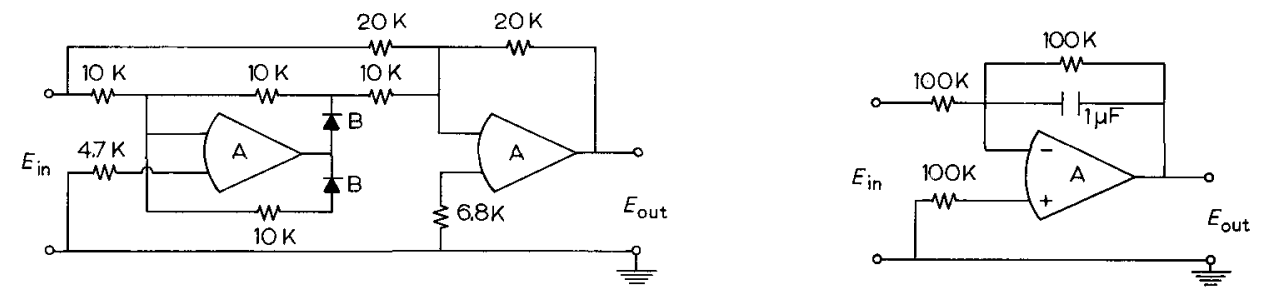

Fig. 3. Full wave rectifier, precision absolute value circuit ${ }^{8}$. (A) Analog devices 118 A, (B) AAZ 15 germanium diodes.

Fig. 4. Leaking integrator. (A) Analog device $118 \mathrm{~A}$.

The use of a tuned amplifier to amplify the a.c. signal of twice the initial frequency caused by the faradaic reaction, suppresses noise and other interfering signals. After the fall of a drop an overload occurred, caused by the fact that the potentiostat is adjusted to the ohmic resistance pertaining to the end of drop-life and consequently miscompensates at the beginning of drop-life.

In order to avoid large oscillations on the recorder, the output of the tuned amplifier was led through the circuit in Fig. 4. The integrating capacitor Cand the "leaking" resistance $\mathrm{R}$ were chosen so that their RC-time was large compared to the reciprocal of the frequency, but small compared to the drop-time. In this way a damped signal was obtained with a magnitude close to the maximal current occurring just before the mercury drop dislodged.

Throughout all experiments an oscilloscope was used to monitor the output of the several parts of the total circuit.

RESULTS

Figure 5 shows oscilloscopic traces of the capacity current respectively before rectification (a) and after rectification (b). Trace (c) gives the sum of capacity and faradaic current before rectification and curve (d) the same after rectification. It is clear that the capacity current gives rise only to a d.c. level superimposed by some noise, whereas the faradaic current has a substantial wave form similar to the theoretical pattern shown in Fig. 2c, III. 


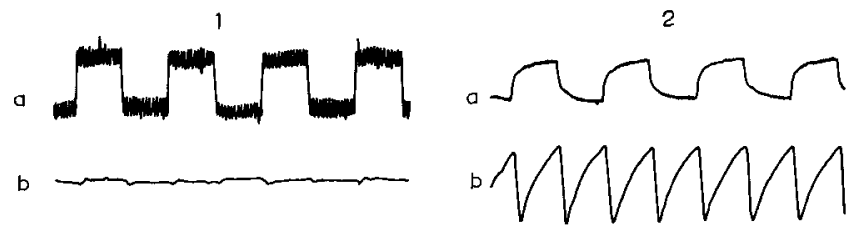

Fig. 5. Oscilloscopic traces of base line current (1) and peak current (2) for $\mathrm{Cd}^{2+}$ in $1 \mathrm{M} \mathrm{KCl}$. (a) Before rectification, (b) after rectification.

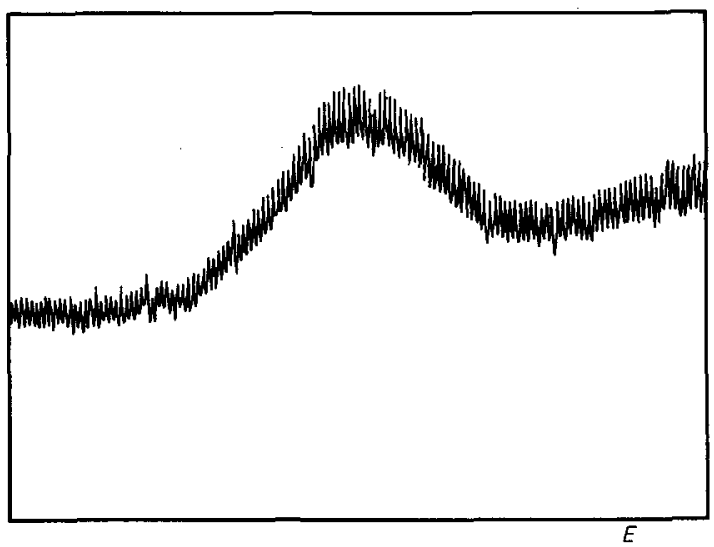

Fig. 6. Polarogram of $5 \times 10^{-6} M \mathrm{Cd}^{2+}$ in $1 M \mathrm{KCl}$ at $210 \mathrm{~Hz}$.

A typical polarogram of $5 \times 10^{-6} \mathrm{M} \mathrm{Cd}^{2+}$ in $1 \mathrm{M} \mathrm{KCl}$ is reproduced in Fig. 6 . A substantial peak is evident. The base line current is not zero, contrary to theoretical expectations. This must be ascribed to non-ideality of the electrical set-up, e.g. as concerns the precision of the triangular wave form injected and the noise introduced by the potentiostat. Also the accuracy with which the potentiostat is adjusted plays an important role. If overcompensation is made, the system starts to oscillate, which is seen as large overloads. Therefore the potentiostat never can compensate the ohmic resistance completely and consequently the capacity current does not attain the ideal square-wave form. This can be seen in Fig. 5, where a significant rise time is observed in trace (a).

That the capacity current is lowered to an appreciable extent is shown in Fig. 7, where a normal sine-wave a.c. polarogram is compared with a polarogram obtained with our set-up. Especially at less negative potentials, where the doublelayer capacity is large, a considerable improvement is obtained.

Since for a reversible electrode reaction the peak height can be expected to increase with frequency, according to eqns. (20) and (21), it is worthwhile to consider the peak height (i.e. peak current minus base current) in relation to the base line current, in order to sort out the optimum frequency. A survey is given in Table 2.

Indeed the peak height is found to increase up to a maximum at $365-415 \mathrm{~Hz}$. This maximum and the decrease after it are caused by the fact that the residual capacity current, due to non-ideal behavior of the set-up, and the faradaic current are added 


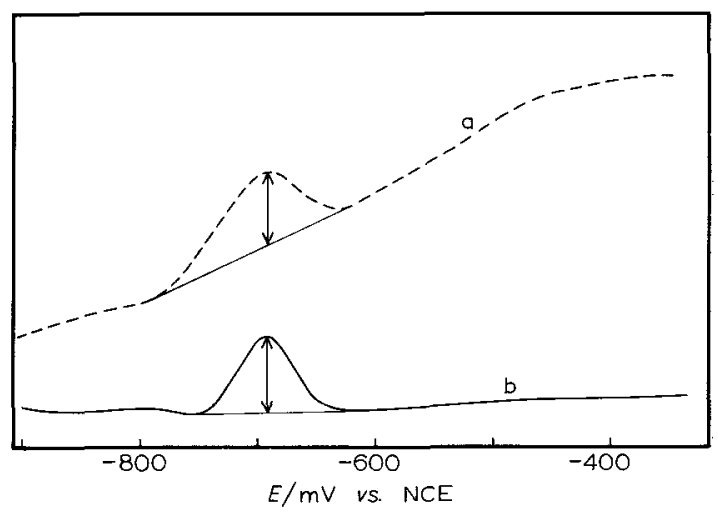

Fig. 7. A.c. polarograms of $2 \times 10^{-5} M \mathrm{Cd}^{2+}$ in $1 M \mathrm{KCl}(50 \mathrm{~Hz})$. (a) Normal sine wave polarogram, (b) triangular wave polarogram with current rectification $(65 \mathrm{~Hz})$.

\section{TABLE 2}

$0.01 \mathrm{~m} M \mathrm{Cd}^{2+}$; A.C. VOLTAGE $20 \mathrm{mV}$; CURRENTS IN ARBITRARY UNITS

\begin{tabular}{lccc}
\hline Frequency/Hz & Base current & Peak current & Ratio \\
\hline 65 & 35 & 55 & 1.58 \\
115 & 53.5 & 100 & 1.87 \\
$165^{\circ}$ & 77.5 & 165 & 2.13 \\
215 & 130 & 198 & 1.52 \\
265 & 196 & 202 & 1.04 \\
315 & 262 & 220 & 0.84 \\
365 & 350 & 248 & 0.71 \\
415 & 437 & 240 & 0.55 \\
465 & 545 & 245 & 0.45 \\
530 & 645 & 155 & 0.24 \\
590 & 785 & 165 & 0.21 \\
720 & 1200 & 180 & 0.15 \\
910 & 1880 & 150 & 0.08 \\
\hline
\end{tabular}

vectorially, while there is a phase shift between the two. As in ordinary a.c. polarography the frequency at which the maximum occurs depends on the concentration and the reversibility of the electroactive species and has no direct physical meaning.

The optimum ratio of peak height to base line is found at $165 \mathrm{~Hz}$. At this frequency and at $210 \mathrm{~Hz}$, which is near to it, a calibration was made by running polarograms for various $\mathrm{Cd}^{2+}$ concentrations. Straight calibration lines were found up to $2 \times 10^{-4} M \mathrm{Cd}^{2+}$. At higher concentrations a slight bending downwards is observed, as is usual in a.c. polarography, probably caused by faulty compensation of the ohmic resistance. The lower concentration parts of the calibration lines are shown in Fig. 8. They show the peculiar feature that at concentrations below $2.5 \mu M$ $\mathrm{Cd}^{2+}$, the peak height is actually zero. It seems that at all concentrations an equal amount of the faradaic current is "lost". We did not find an explanation for this phenomenon, but we are sure that it originates from the inadequacy of our electrical set-up, because it also occurred with a dummy cell. 

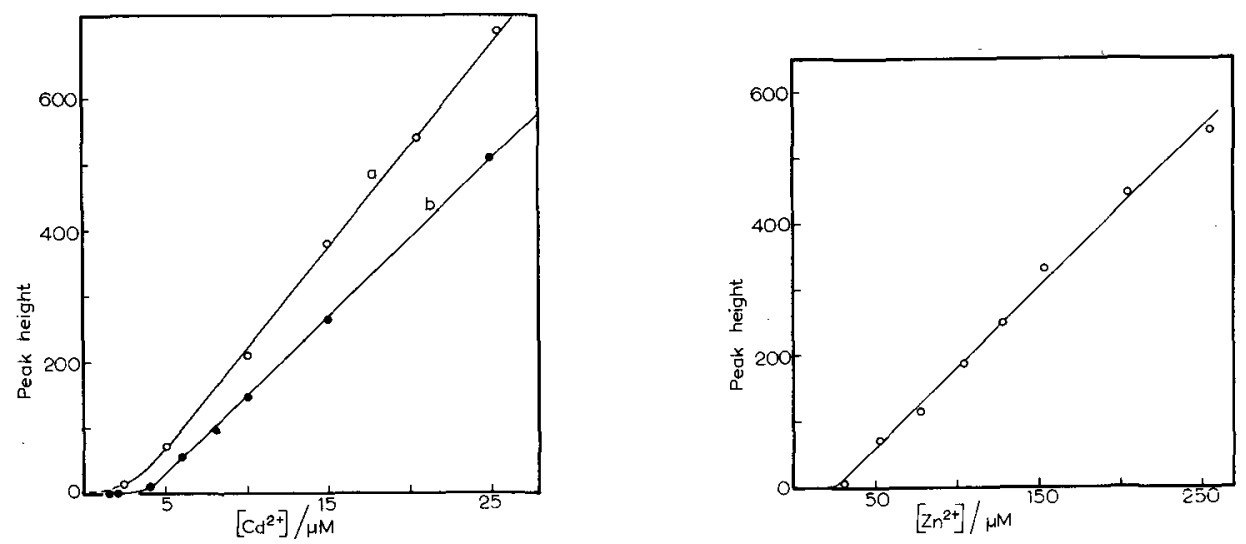

Fig. 8. Calibration line for $\mathrm{Cd}^{2+}$ in $1 M \mathrm{KCl}$ at: (a) 210 , (b) $160 \mathrm{~Hz}$.

Fig. 9. Calibration line for $\mathrm{Zn}^{2+}$ in $1 \mathrm{M} \mathrm{KCl}$ at $210 \mathrm{~Hz}$.

That the effect has no chemical origin (e.g. loss of $\mathrm{Cd}^{2+}$ by hydrolysis) is also proved by the calibration line for $\mathrm{Zn}^{2+}$ in $1 \mathrm{M} \mathrm{KCl}$, drawn in Fig. 9. Since this system behaves almost irreversibly, its amplitude is smaller than for $\mathrm{Cd}^{2+}$ and consequently the lowest accessible concentration is $25 \mu \mathrm{M}$ instead of $2.5 \mu \mathrm{M}$. However, the same loss of signal is still observed.

It is rather difficult to compare the merits of this new polarograph with other polarographic techniques. Several aspects should be taken into account such as the price of the equipment, the definition of sensitivity, detection limit, and accuracy, the aim of the analysis, etc. We prefer to confine ourselves to the fact that our design can be built from rather inexpensive accessories. The accuracy in the recorder reading may be estimated to be $1 \%$. Regarding Table 2 it can be concluded that at the optimum frequency a concentration of $5 \times 10^{-7} \mathrm{M} \mathrm{Cd}^{2+}$ should produce a peak height which is still $10 \%$ of the base line current. Therefore, if the loss of signal mentioned before is eliminated, this can be considered as the detection limit of the apparatus. From the calibration lines in Fig. 8 it can be seen that concentrations exceeding $5 \times 10^{-6} M$ can easily be determined with $1 \%$ accuracy. This limit is $c a .20$ times better than normal a.c. polarography.

\section{ACKNOWLEDGEMENT}

This investigation was supported in part by the Netherlands Foundation for Chemical Research (S.O.N.) with financial aid from the Netherlands Organization for the Advancement of Pure Research (Z.W.O.).

\section{SUMMARY}

It is proposed to eliminate the influence of the double layer capacity in a.c. polarography by applying a triangular voltage instead of the usual sine wave voltage. In the case of the triangular voltage the capacity current is a square wave that can be 
eliminated after full-wave rectification and filtering the resulting direct voltage. Any faradaic current is shown to keep an a.c. component after this operation. After a discussion of the theory, preliminary experiments are reported.

\section{REFERENCES}

1 B. Breyer and F. Gutmann, Australian J. Sci., 8 (1945) 21, 163; Trans. Faraday Soc., 42 (1946) 645, 650; 43 (1947) 785; Discussions Faraday Soc., 1 (1947) 19.

2 G. JEssop, British patent 640,768 (1950).

3 G. Jessop, British patent 776,543 (1957).

4 G. C. Barker AND J. L. JenKins, Analyst, 77 (1952) 685.

5 H. Eyring, S. Glasstone and K. J. Laidler, J. Chem. Phys., 7 (1939) 1053.

6 M. Sluyters-Rehbach and J. H. SluYters in A. J. Bard (Ed.), Electroanalytical Chemistry, Vol. 4, Marcel Dekker, New York, 1970, p. 1.

7 E. R. Brown, D. E. Smith and G. L. Booman, Anal. Chem., 40 (1968) 1141.

8 Burr-Brown, Handbook of Operational Amplifier Applications, private edition of Burr-Brown Factory.

J. Electroanal. Chem., 31 (1971) 201-210 